\title{
Correction to: Tumor-suppressive effects of atelocollagen-conjugated hsa-miR-520d-5p on un-differentiated cancer cells in a mouse xenograft model
}

Yoshitaka Ishihara', Satoshi Tsuno', Satoshi Kuwamoto², Taro Yamashita ${ }^{3}$, Yusuke Endo ${ }^{1}$, Keigo Miura ${ }^{4}$, Yugo Miura ${ }^{5}$, Takemasa Sato ${ }^{6}$, Junichi Hasegawa ${ }^{1}$ and Norimasa Miura ${ }^{1 *}$

\section{Correction}

After publication of the original article [1] the authors found the following errors had occurred:

1. The imaging photo was incorrectly selected for Fig. 4. The original article contains imaging relating to another series. An updated version of Fig. 4 is included with this Correction.

2. In the original Fig. 5b figure legend, reference is made to an HE stain, however this has been made incorrectly. All references to an HE stain should therefore be omitted. The entirety of Figure legend $5 \mathrm{~b}$ is included below, with the amendments made to the first sentence (which is included in bold for reference):

\section{Figure legend 5b:}

Microscopic examination of HLF (left; $\times 100$ magnification) and HMV-I tumors (right; $\times 200$ magnification) that displayed suppressed tumor growth. HLF tumors treated with 520d/atelocollagen exhibited GFPexpressing muscle tissues, including undifferentiated tumor cells unaccompanied by necrosis. HMV-I tumors treated with $520 \mathrm{~d} /$ atelocollagen showed undifferentiated neoplastic cells. This finding is not inconsistent, as malignant melanomas are observed to be accompanied by extensive necrosis, as shown around A.

A corrected version of Fig. 5 is included with this Correction.

\begin{abstract}
Author details
'Division of Pharmacotherapeutics, Department of Pathophysiological \& Therapeutic Science, Faculty of Medicine, Tottori University, 86 Nishicho, Yonago, Tottori 683-8503, Japan. ${ }^{2}$ Division of Molecular Pathology, Faculty of Medicine, Tottori University, 86 Nishicho, Yonago, Tottori 683-8503, Japan. ${ }^{3}$ Department of Gastroenterology, Tottori University Hospital, 86 Nishicho, Yonago, Tottori 683-8504, Japan. ${ }^{4}$ PEZY-Pharma, 86 Nishicho, Yonago, Tottori 683-8503, Japan. ${ }^{5}$ Orthopedic Surgery, Tokyo Medical and Dental University, 1-5-45 Yushima, Bunkyo-ku, Tokyo 113-8510, Japan. ${ }^{6}$ Division of Neurobiology, School of Life Science, Faculty of Medicine, Tottori University, 86 Nishicho, Yonago, Tottori 683-8503, Japan.
\end{abstract}

Received: 25 September 2017 Accepted: 25 September 2017 Published online: 02 October 2017

\section{Reference}

1. Ishihara $Y$, Tsuno S, Kuwamoto S, Yamashita T, Endo Y, Miura K, Miura $Y$, Sato T, Hasegawa J, Miura N. Tumor-suppressive effects of atelocollagenconjugated hsa-miR-520d-5p on undifferentiated cancer cells in a mouse xenograft model. BMC Cancer. 2016;16:415. doi:10.1186/s12885-016-2467-y.

* Correspondence: mnmiura@med.tottori-u.ac.jp

${ }^{1}$ Division of Pharmacotherapeutics, Department of Pathophysiological \&

Therapeutic Science, Faculty of Medicine, Tottori University, 86 Nishicho,

Yonago, Tottori 683-8503, Japan

Full list of author information is available at the end of the article 


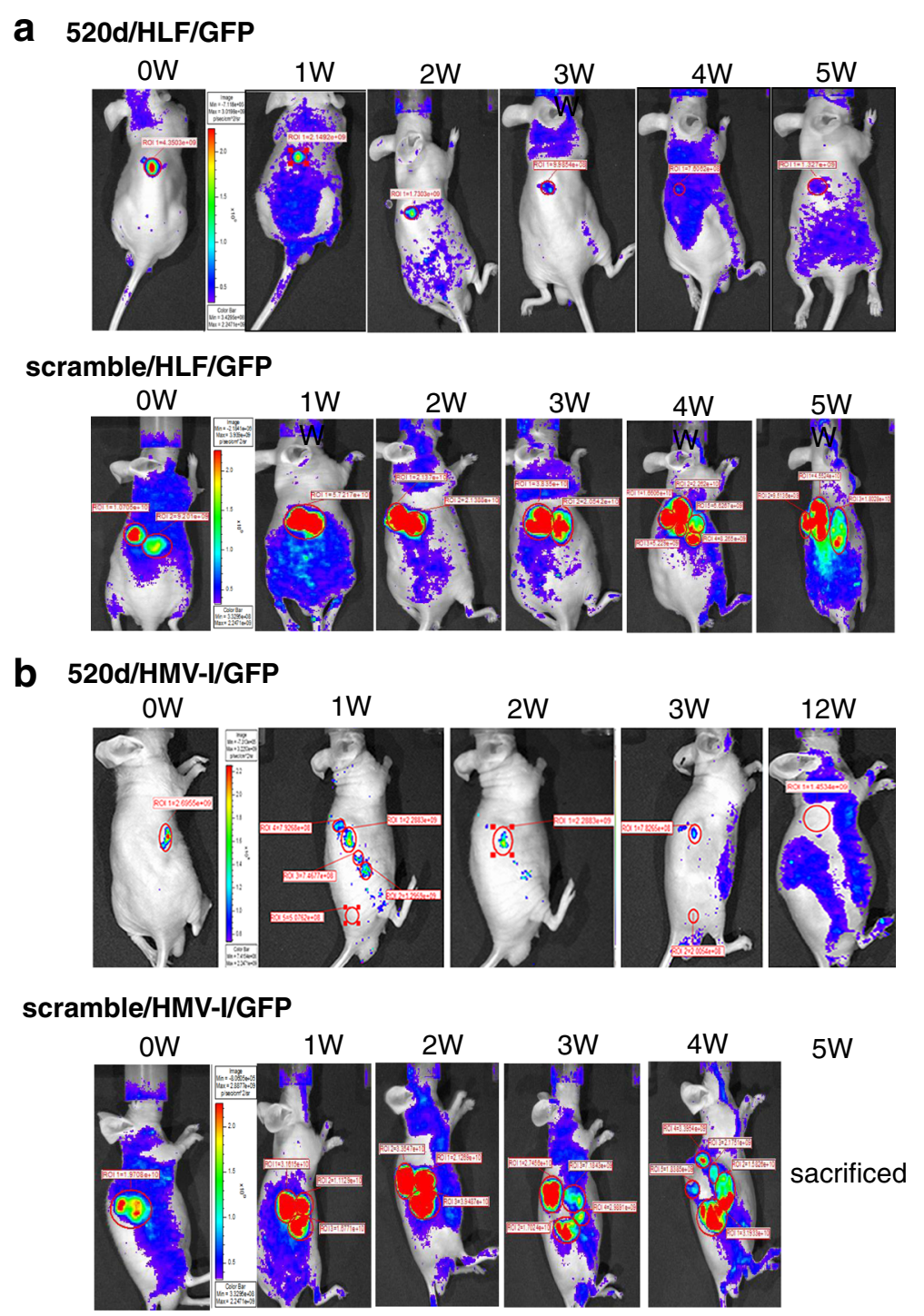

Fig. 4 The therapeutic effect of 520d-5p-conjugated atelocollagen on subcutaneously inoculated tumors as monitored by an in vivo imaging system. a A representative image of the therapeutic effect of 520d-5p/atelocollagen using an in vivo imaging system. At each inoculation site, 37.5\% (3/8) of the tumors disappeared. The growth of tumors that received $520 \mathrm{~d}-5 \mathrm{p}$ was significantly suppressed, and metastatic ability was entirely inhibited in the remaining mice. A time course (top) of 520d/HLF/GFP cells (520d-5p-transfected and GFP-expressing HLF) is depicted. Representative sequential images (bottom) of scrambled/HLF/GFP cells (scrambled-transfected GFP-expressing HLF). b In HMV-I tumors, 12.5\% (1/8) of the tumors disappeared. After treatment with 520d-5p, the growth of other tumors was significantly suppressed, and their metastatic ability was entirely inhibited. 520d/HMV-I/GFP and scrambled/ HMV-I/GFP indicate 520d-5p-transfected, GFP-expressing HLF (top) and scramble-transfected, GFP-expressing HMV-I (bottom), respectively 


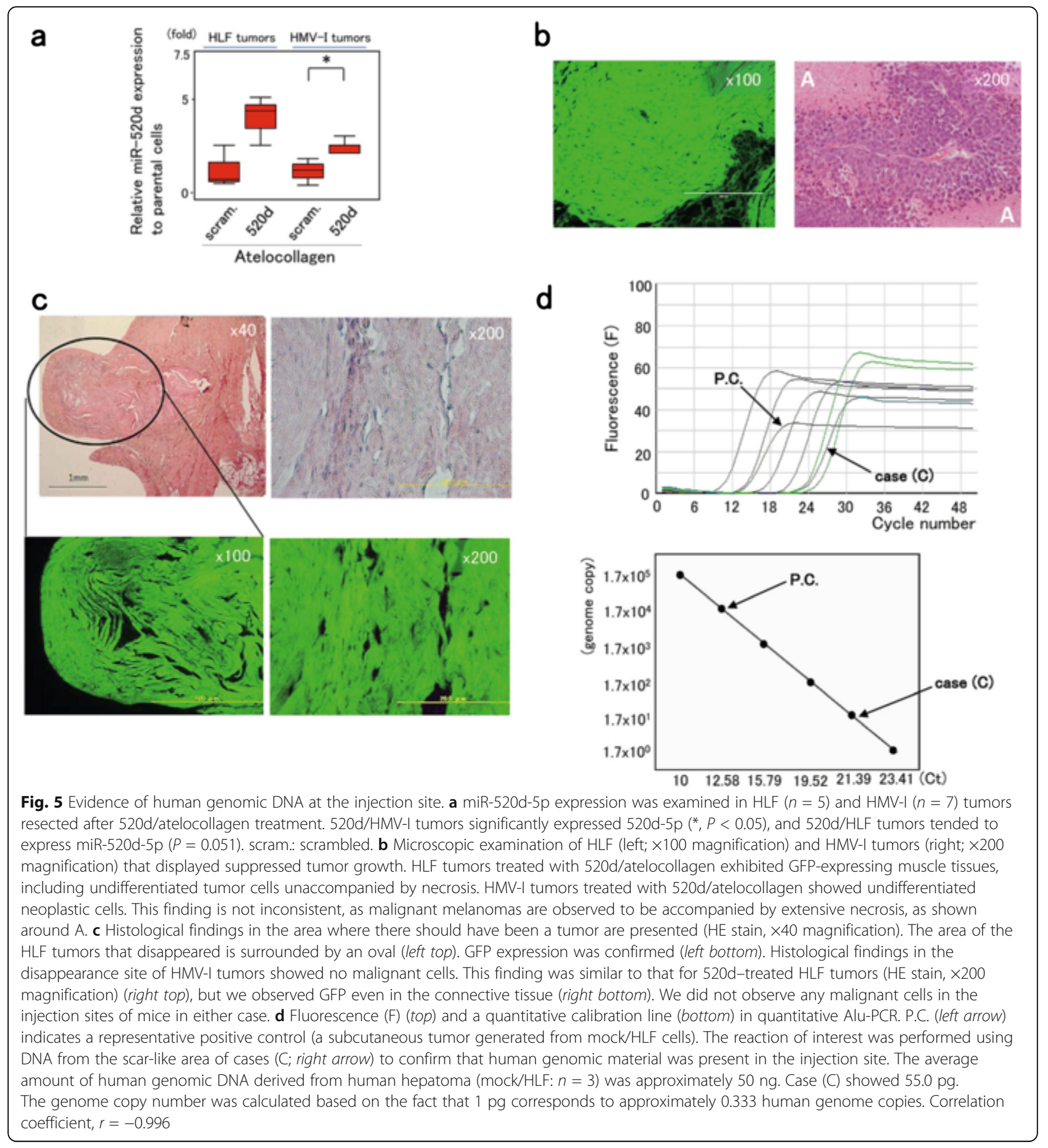

